\title{
Advanced Copy Propagation for Arrays
}

\author{
Peter Vanbroekhoven ${ }^{*}$ \\ Gerda Janssens \\ Maurice Bruynooghe \\ Katholieke Universiteit Leuven \\ Department of Computer Science \\ Celestijnenlaan 200A \\ B-3001 Leuven, Belgium \\ \{peterv,gerda,maurice\}@cs.kuleuven.ac.be
}

\author{
Henk Corporaal \\ Francky Catthoor \\ Interuniversity MicroElectronics Center \\ Kapeldreef 75 \\ B-3001 Leuven, Belgium \\ \{heco,catthoor\}@imec.be
}

\begin{abstract}
The focus of this paper is on a data flow-transformation called advanced copy propagation. After an array is assigned, we can, under certain conditions, replace a read from this array by the righthand side of the assignment. If so, the intermediate assignment can be skipped. In case it becomes dead code, it can be eliminated. Where necessary we distinguish between the different elements of arrays as well as the different runtime instances of statements, allowing us to do propagation over global loop and condition scopes. We have formalized two basic operations: non-recursive propagation that operates on two statements and recursive propagation that operates on one statement. A global algorithm uses these two operations to do propagation on code involving any number of statements. Running our prototype implementation on some multimedia kernels shows that we can get a decrease in memory acesses between $22 \%$ and $43 \%$.
\end{abstract}

\section{Categories and Subject Descriptors}

D.3.4 [Programming Languages]: Processors-Compilers, Optimization

\section{General Terms}

Algorithms, Languages

\section{Keywords}

copy propagation, optimization, single assignment, arrays

\section{INTRODUCTION}

\footnotetext{
${ }^{*}$ Supported by a specialisation grant from the Institute for the Promotion of Innovation by Science and Technology in Flanders (IWT)
}

Permission to make digital or hard copies of all or part of this work for personal or classroom use is granted without fee provided that copies are not made or distributed for profit or commercial advantage and that copies bear this notice and the full citation on the first page. To copy otherwise, to republish, to post on servers or to redistribute to lists, requires prior specific permission and/or a fee.

LCTES'03, June 11-13, 2003, San Diego, California, USA.

Copyright 2003 ACM 1-58113-647-1/03/0006 ...\$5.00.
Since the rise of the World Wide Web, the number of multimedia and network applications has been growing rapidly. These applications process large amounts of data. In e.g., a C-program this data will be stored in large arrays. Techniques exist to allocate arrays to registers[12], but most arrays will still be in memory. This is however a problem because of the exponentially growing gap between processor speed and main memory speed[8]. Another problem with large, high-speed memories is that they consume much power and hence have a large heat dissipation[3]. Current hardware technology is unable to solve this problem.

This is especially a problem for data intensive applications on embedded systems. The solution is to transform these programs such that the memory accesses and the power consumption are reduced. This is exactly the aim of the Data Transfer and Storage Exploration (DTSE) methodology [3]. One of the steps proposed there is to do advanced copy propagation for arrays ${ }^{1}$, and more specifically on programs in dynamic single assignment (DSA) where every variable (thus also every array element) is written only once during the execution of the program. This is a stronger form of single assignment than the well-known static single assignment $[1,12]$. Removing copy operations is important since they occur readily in multimedia applications. This can either be because an operation in the program is inherently a copy operation, like a swap of two array elements or a transposition of a matrix, or because introducing extra copy operations simplifies writing a program or reasoning about a program, like in image processing where a border is added to an image to account for border conditions.

The idea of removing copy operations already exist as copy propagation in the classic compiler literature[1, 12]. The idea there is that after an assignment $f=g$, if possible, $g$ is used instead of $f$. If then the assignment becomes dead code, it can be eliminated. In that case we save two memory accesses and a memory location. Nonetheless, simple copy propagation fails on the first piece of code in Fig. 1. First note that this piece of code can be part of a larger program, the only thing of importance is that there are no further reads from array a, so S4 is the last read from a. We would now like to propagate copy operation S2 to S3. One bump in the road is that the copy propagation from [12] does not take subscripts into account. But even if it did, we cannot just propagate S2 to S3 since elements 0 through 49 read

\footnotetext{
${ }^{1}$ In [3] it is called advanced signal propagation.
} 


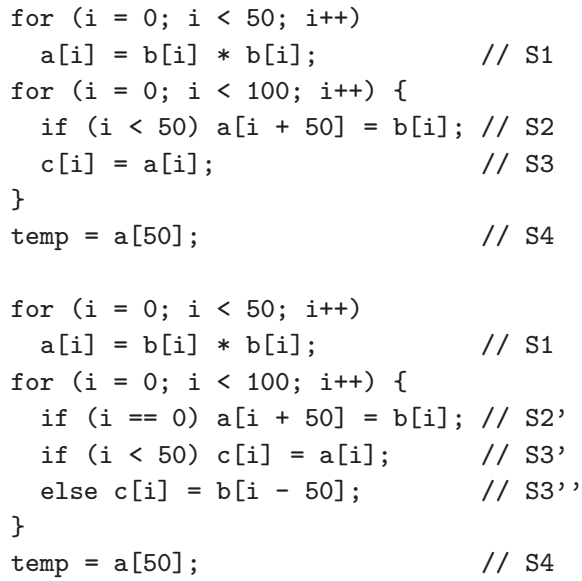

Figure 1: Top: an example on which classic copy propagation fails. Bottom: Propagated S2 to S3 using advanced copy propagation

by $\mathrm{S} 3$ are not written by $\mathrm{S} 2$ but by $\mathrm{S} 1$ which has a different righthand side. Also if we did propagate, S2 would not become dead code yet because S4 still reads a[50]. Yet only a [50] should be written, i.e., S2 is dead code for all iterations except for $i$ equal to 0 .

We show in this paper how to overcome the limitations of classic copy propagation by distinguishing between the different runtime executions of a statement, called instances. For e.g., S2 in Fig. 1 we distinguish between 50 instances, one for each value of iterator i smaller than 50. This allows us to propagate only part of the instances of a statement and then remove only the instances of the statement that have become dead code. Propagating S2 to S3 in the example above, and removing dead code, gives us the second program in Fig. 1. As can be seen, S2' is only executed for $i=0$ and $\mathbf{S} 3$ is split into two statements S3' and S3' ' that are conditionally executed to allow propagation to $\mathrm{S} 3$ ' ' only, as required. The net effect is that 49 instances of S2 are removed and thus 98 memory accesses are removed (49 for a and 49 for $\mathrm{b}$ ). To do this we have to introduce extra conditions and there is some code duplication, but executing these is less expensive than memory accesses as far as power consumption is concerned. Also note that elements 51 through 99 are no longer accessed, so the array can be shrunk which decreases power consumption some more since smaller memories use less power.

The mathematical methods presented here are not limited to copy propagation. They can be used to do "advanced constant propagation" and "advanced expression propagation" as well. However we limit ourselves in this paper to advanced copy propagation since expression propagation in general can increase the number of memory accesses.

To be able to easily distinguish between the runtime instances of statements, we apply our methods to programs in dynamic single assignment (DSA) form. Since array elements are then assigned a value only once, they become equivalent to the values they were assigned, allowing much easier transformations on the data flow. Currently only a subset of imperative programs can be automatically converted to DSA form[6, 9]. Fortunately many multimedia kernels belong to that subset. Hence DSA is already widely used in the context of parallelization [7] and systolic array

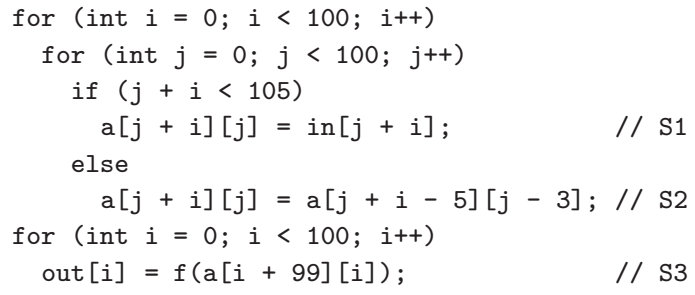

Figure 2: Our running example

design [11]. We are currently working on a new method that will be able to extend the subset of programs that can be transformed to DSA. A second issue is that array sizes can grow considerably. Advanced methods exist for compacting arrays $[5,17]$. When used in combination with loop transformations[4], they can reduce the arrays again - often beyond their original size.

In Sec. 2 we explain how we geometrically model the subset of programs we can handle. Advanced copy propagation is explained in Sec. 3 : Sec. 3.1 contains the basic idea, Sec. 3.2 non-recursive copy propagation, Sec. 3.3 recursive copy propagation and Sec. 3.4 wraps up the running example. Finally we present some results in Sec. 4, a few aspects of the use of DSA in Sec. 5 and a comparison of our method to related work in Sec. 6.

\section{PRELIMINARIES}

The data-flow transformations presented in this paper can handle programs that satisfy the following requirements:

- The program consists of a nest of for-loops and ifstatements. Any possible nesting is allowed. The step of the for-loops should be a constant and is not restricted to 1 .

- Anywhere in the nesting, assignment statements can be present. The lefthand side of an assignment is to an element of an array. ${ }^{2}$ The righthand side of the assignment can be any expression containing array references with no restrictions on indexing.

- All expressions used as bounds for for-loops or as tests for if-statements are affine combinations of the surrounding loop iterators, i.e., a linear combination of them plus a constant.

- The program is in DSA form. We demand that the indexation in the lefthand side of an assignment surrounded by $n$ for-loops is of the following form:

$$
A \cdot \vec{\imath}+\vec{c} .
$$

Here $A$ is a non-singular $n \times n$ matrix, $\vec{\imath}$ is a vector containing the iterators of the surrounding loops and $\vec{c}$ is any column vector of length $n$. Note that this is often the case in DSA programs, e.g., the ones that are produced by the DSA conversion in [6].

This is a well-known set of programs, both in the area of parallelization as well as the area of hardware synthesis [16], since these kinds of programs can be modeled and operated on using well-established mathematical methods. The example from Fig. 2 satisfies all of the conditions above.

${ }^{2}$ Note that scalar variables like integers could be considered as arrays of length 1 . 
In this paper the programs are represented by a (notationally) simplified version of the geometrical modeling of [3]. We will introduce our notation using the program in Fig. 2. In that program, there are two loop nests. The first one consists of two loops with iterators $i$ and $j$. The body of this loop nest is executed for different combinations of integral values of $i$ and $j$. We can represent each of these combinations as a point in a two-dimensional iteration space. The set of those points forms the iteration domain for the body of the loop and can be written as

$$
\left\{(i, j) \mid 0 \leq i<100 \wedge 0 \leq j<100 \wedge(i, j) \in \mathbb{Z}^{2}\right\} .
$$

However statement S1 is not executed for all points in this iteration domain, but only for those points $(i, j)$ for which $j+i<105$ is true. So the iteration domain for that statement is

$$
\begin{aligned}
I_{1}=\{(i, j) \mid & 0 \leq i<100 \wedge 0 \leq j<100 \\
& \left.\wedge j+i<105 \wedge(i, j) \in \mathbb{Z}^{2}\right\}
\end{aligned}
$$

in which the subscript refers to the number of the statement. For statement $\mathbf{S} 2$ which is only executed if $j+i<105$ is not true, the iteration domain is

$$
\begin{aligned}
I_{2}=\{(i, j) \mid & 0 \leq i<100 \wedge 0 \leq j<100 \\
& \left.\wedge j+i \geq 105 \wedge(i, j) \in \mathbb{Z}^{2}\right\} .
\end{aligned}
$$

The iteration domain for $\mathrm{S} 3$ is one-dimensional because it has only one surrounding for-loop:

$$
I_{3}=\{(i) \mid 0 \leq i<100 \wedge i \in \mathbb{Z}\} .
$$

Now it is possible to refer to each instance of e.g., statement $\mathrm{S} 1$ as $\mathrm{S} 1\left(\vec{\imath}_{1}\right)$ with $\vec{\imath}_{1} \in I_{1}$, or as $\mathrm{S} 1(i, j)$ with $(i, j) \in I_{1}$. $\mathrm{S} 1(i, j)$ with $(i, j) \in I_{1}$ writes to an element of array a indicated by $w_{1}(i, j)$. In our example this is

$$
w_{1}: \mathbb{Z}^{2} \rightarrow \mathbb{Z}^{2}:(i, j) \mapsto(j+i, j) .
$$

This definition mapping is from a two-dimensional iteration domain to a two-dimensional variable domain. Also S1 $(i, j)$ reads from an element of array in indicated by $r_{1}(i, j)$. In our case $r_{1}$ is specified by

$$
r_{1}: \mathbb{Z}^{2} \rightarrow \mathbb{Z}:(i, j) \mapsto(j+i)
$$

This is an operand mapping from a two-dimensional iteration domain to a one-dimensional variable domain. If there are multiple reads in a single statement, we can distinguish between the operand mappings by adding an extra index. We will not need this facility in this paper.

An important point is that all definition mappings $w_{s}$ are invertible. Since the program is in DSA form, there is a oneto-one mapping between the points in the iteration domain of a statement and the elements written by that statement. Such a one-to-one mapping is always invertible.

The set of elements of an array that are read or written are respectively called operand and definition domain. For $\mathrm{S} 1$ the definition domain is given by:

$$
W_{1}=w_{1}\left(I_{1}\right)=\left\{(a, b) \mid \exists(i, j) \in I_{1}:(a, b)=w_{1}(i, j)\right\} .
$$

Filling in the specifics of statement $\mathrm{S} 1$ gives

$$
\begin{array}{r}
W_{1}=\left\{(a, b) \mid \exists(i, j) \in \mathbb{Z}^{2}: b=j \wedge a=j+i \wedge\right. \\
0 \leq i<100 \wedge 0 \leq j<100 \wedge j+i<105\} .
\end{array}
$$

The iteration and definition domains for $\mathrm{S} 1$ and $\mathrm{S} 2$ are schematically represented in Fig. 3. Similar specifications can be

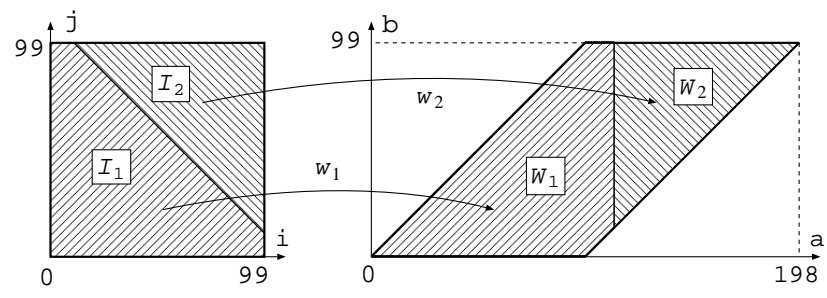

Figure 3: Schematical representation of the iteration domains (left) and definition domains (right) for $\mathrm{S} 1$ and S2

given for operand domains $R_{i}$ of statements $\mathrm{S}_{i}$.

Since we limited the expressions for the bounds of the loops to affine expressions of surrounding iterators, the iteration domains for each statement can be described as the integer points in an $n$-dimensional polyhedron, which is a part of n-dimensional space bounded by linear inequalities. Here $n$ is the number of loops around the statement in question. In case the step of the for-loops is not 1 , an extension of this, called $\mathbb{Z}$-polyhedra, is used. Finding definition and operand domains is then the image of a $\mathbb{Z}$-polyhedron by an invertible, affine mapping, which in turn is a $\mathbb{Z}$-polyhedron itself. Defining $\mathbb{Z}$-polyhedra and doing operations on them like image through an affine mapping or set operations like intersection can be handled by a polyhedral library as in [15]. An important feature of this library is that a conjunction of affine conditions on iterators can be simplified by discarding all conditions entailed by the bounds on the iterators.

In the remainder of the paper we will use the representation from this section together with the equivalent $\mathrm{C}$ program. Note that the methods in this paper can be applied to any language, e.g., Fortran, as long as it has arrays and for-loops.

\section{COPY PROPAGATION METHOD}

This section first describes automatable operations necessary to do copy propagation over global loop and condition scopes. The arrays we are interested in are those that are copies of other arrays. The idea is to replace reads from such copy arrays by reads from the copied arrays. The effect of this is that (part of) the copy is no longer read and thus can be removed, along with the corresponding copy operations.

We first explain the basic idea behind advanced copy propagation in Sec. 3.1. This idea is then elaborated in two basic operations: propagation of a statement that copies values written by another statement (Sec. 3.2) and propagation of a statement that copies values it wrote itself (Sec. 3.3). We apply these basic operations to an example and we finish off this example in Sec. 3.4.

\subsection{Basic idea}

In the example of Fig. 2, statements $\mathrm{S} 1$ and $\mathrm{S} 2$ write to array a, and statements S2 and S3 read from it. In Fig. 4 the definition and operand domains related to a are represented. This figure is the same as the right side of Fig. 3, but with the operand domains added as the two shaded areas. We see that R2 overlaps with W1 and W2, which means that statement $\mathrm{S} 2$ reads array elements written by both $\mathrm{S} 1$ and itself. We also see that R3 overlaps with W1 and W2, meaning that S3 reads array elements written by $\mathrm{S} 1$ and $\mathrm{S} 2$. 


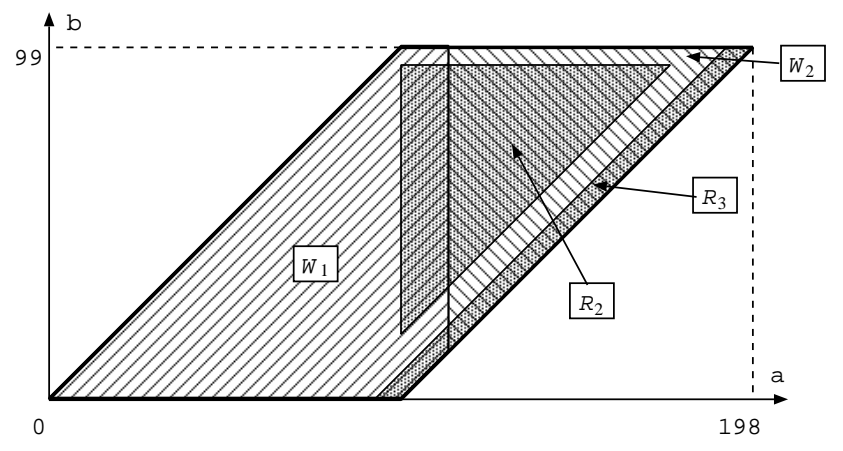

Figure 4: Schematical representation of definition and operand domains for statements related to a

Since S2 reads array elements written by S1, we will try to replace the read from array a in statement $\mathrm{S} 2$ by the righthand side of $\mathrm{S} 1$, with the correct values for $i$ and $j$. We need to find out which $\mathrm{S} 1\left(i_{w}, j_{w}\right)$ wrote the array element read by $\mathrm{S} 2\left(i_{r}, j_{r}\right)$. If $\mathrm{S} 2\left(i_{r}, j_{r}\right)$ is to read the value written by $\mathrm{S} 1\left(i_{w}, j_{w}\right)$, then they should refer to the same array element, i.e., $r_{2}\left(i_{r}, j_{r}\right)=w_{1}\left(i_{w}, j_{w}\right)$. Recall that $w_{1}$ should be invertible. So we can then find $i_{w}$ and $j_{w}$ as follows:

$$
\left(i_{w}, j_{w}\right)=\left(w_{1}^{-1}\right)\left(r_{2}\left(i_{r}, j_{r}\right)\right) .
$$

We applied $w_{1}^{-1}$, a function in two arguments, to a single couple, meaning that each of the elements of the couple are passed as arguments to $w_{1}^{-1}$ in the same order as they appear in the couple. Then in statement $\mathrm{S} 2\left(i_{r}, j_{r}\right)$ we can replace the read from the array element indicated by $r_{2}\left(i_{r}, j_{r}\right)$ by the righthand side of $\mathrm{S} 1\left(\left(w_{1}^{-1}\right)\left(r_{2}\left(i_{r}, j_{r}\right)\right)\right)$. This however is only possible if $\mathrm{S} 1\left(\left(w_{1}^{-1}\right)\left(r_{2}\left(i_{r}, j_{r}\right)\right)\right)$ is actually executed and this is when

$$
\left(w_{1}^{-1}\right)\left(r_{2}\left(i_{r}, j_{r}\right)\right) \in I_{1} .
$$

Thus we can only propagate the array written by $\mathrm{S} 1$ to S2 $\left(i_{r}, j_{r}\right)$ when (11) is true, otherwise we need to continue using the original array reference.

The transformation above is always valid on a program in DSA form, so no extra conditions need to be checked. This is unlike classic copy propagation that is only allowed under certain conditions. In the case of a DSA program, these conditions are automatically satisfied. We know for a fact that assignment $\mathrm{S} 1\left(\left(w_{1}^{-1}\right)\left(r_{2}\left(i_{r}, j_{r}\right)\right)\right)$ is the only assignment to the memory element read, and hence it can be the only definition of the array element that reaches the read. Also if we suppose the program is correct, than this single definition of the array element must have happened before the read from it. A less obvious condition is that the array element indicated by $r_{2}\left(i_{r}, j_{r}\right)$ should have the same value when $\mathrm{S} 2\left(i_{r}, j_{r}\right)$ is executed and when $\mathrm{S} 1\left(\left(w_{1}^{-1}\right)\left(r_{2}\left(i_{r}, j_{r}\right)\right)\right)$ is executed, but since in a correct DSA program the single assignment to the array element must have happened before either of them, this condition is fulfilled too.

Once we have propagated $\mathrm{S} 1$ to $\mathrm{S} 2$, some instances of $\mathrm{S} 1$ may have become dead code and hence we can remove them. Let $S=\left\{\mathrm{S}_{i_{1}}, \ldots, \mathrm{S}_{i_{s}}\right\}$ be the set of other statements reading values written by $\mathrm{S} 1$. With $R_{i_{j}}$ the operand domain of $\mathrm{S}_{i_{j}}$, the set of array elements read by $S$ is given by $R=R_{i_{1}} \cup$ $\ldots \cup R_{i_{s}}$. Hence $\mathrm{S} 1$ still has to be executed for the iterations

$$
w_{1}^{-1}(R) \cap I_{1} \text {. }
$$

The intersection with $I_{1}$ is necessary as statements different from $\mathrm{S} 1$ can write some of the elements in $R$.

\subsection{Non-recursive copy propagation}

In this section we show how to apply the basic idea of Sec. 3.1 to a statement that does not read any array elements that it wrote itself. The case where a statement does read values it wrote itself is handled in Sec. 3.3. The case we consider in this section is either when a statement reads from different arrays than it writes to, or when it reads from another part of the same array than where it writes to. Formally, the latter means that for statement $\mathrm{S}_{x}, W_{x} \cap R_{x}=\emptyset$.

In the program from Fig. 2, statement $\mathrm{S} 1$ falls into this category since it reads from array in and writes to array a. We will propagate the array elements written by $\mathrm{S} 1$ to $\mathrm{S} 2$, i.e., we will replace the read from array a in S2 by the righthand side of $\mathrm{S} 1$. We first need to calculate (10). We know $r_{2}$, so we only need to calculate $w_{1}^{-1}$ :

$$
w_{1}^{-1}: \mathbb{Z}^{2} \rightarrow \mathbb{Z}^{2}:(x, y) \mapsto(x-y, y) .
$$

Equation (10) then becomes

$$
\left(i_{w}, j_{w}\right)=\left(w_{1}^{-1}\right)\left(j_{r}+i_{r}-5, j_{r}-3\right)=\left(i_{r}-2, j_{r}-3\right) .
$$

Substituting (14) in the righthand side of $\mathbf{S} 1\left(i_{w}, j_{w}\right)$ then gives:

$$
\operatorname{in}\left[i_{r}+j_{r}-5\right]
$$

This is the expression we want to substitute into $\mathrm{S} 2\left(i_{r}, j_{r}\right)$. The condition under which this is allowed is given by (11):

$$
\left(i_{r}-2, j_{r}-3\right) \in I_{1} .
$$

Filling in (3) for $I_{1}$, we get

$$
0 \leq i_{r}-2<100 \wedge 0 \leq j_{r}-3<100 \wedge j_{r}+i_{r}-5<105 .
$$

Note that we left out the condition that $\left(i_{r}-2, j_{r}-3\right)$ should be in $\mathbb{Z}^{2}$ because this is always the case since $i_{r}$ and $j_{r}$ are integer iterators. However in this case we can simplify this condition even more. Any element of array a is written by either $\mathbf{S} 1$ or $\mathbf{S} 2$, as can easily be derived from Fig. 2. The condition under which $\mathrm{S} 1\left(i_{w}, j_{w}\right)$ wrote the value read by $\mathrm{S} 2\left(i_{r}, j_{r}\right)$ is given in (16). In the same way the condition under which $\mathrm{S} 2\left(i_{w}, j_{w}\right)$ wrote the value read by $\mathrm{S} 2\left(i_{r}, j_{r}\right)$ is the following:

$$
\left(i_{r}-2, j_{r}-3\right) \in I_{2} .
$$

Note that this is nearly the same as (16). This is not surprising since both definition mappings, i.e., $w_{1}$ and $w_{2}$, are the same. Filling in (4) for $I_{2}$, we get

$$
0 \leq i_{r}-2<100 \wedge 0 \leq j_{r}-3<100 \wedge j_{r}+i_{r}-5 \geq 105 .
$$

We see that (17) and (19) have a part in common, in particular the part that refers to the loop bounds. This is not surprising either since the two statements have two loops in common. Either (17) or (19) has to be true - an array element, if read, should be written by some statement and this means that the common part is always true. This supposes of course that the program shown is a complete program and no other assignments to a are present. Hence we can leave this part of the condition out. Equation (17) can thus be simplified to:

$$
j_{r}+i_{r}-5<105 \Leftrightarrow j_{r}+i_{r}<110 .
$$




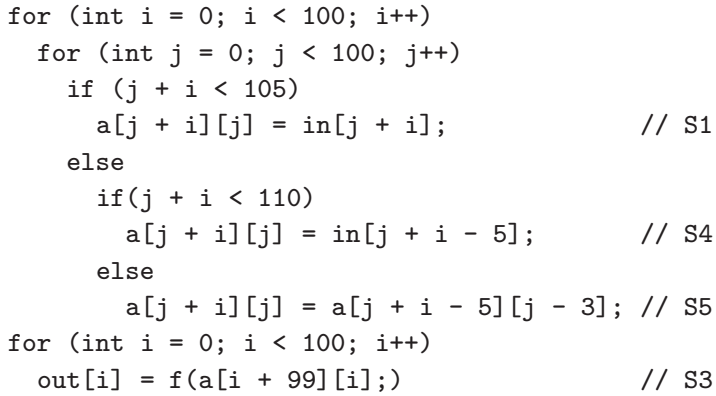

Figure 5: S1 has been propagated to S2

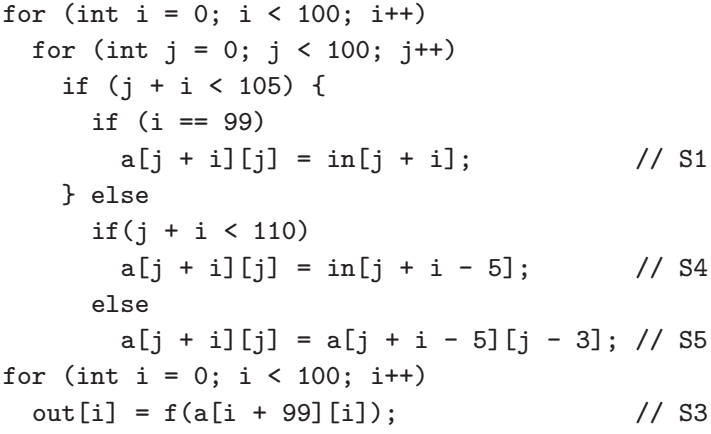

Figure 6: Dead instances of S1 removed

Now we have all information to actually do the propagation on the example. The result is the program in Fig. 5. Notice that statement S2 has been split up in two statements, S4 and S5, and that an if-statement has been added such that $\mathrm{S} 4\left(i_{r}, j_{r}\right)$ is only executed if $(20)$ is true, while $\mathrm{S} 5\left(i_{r}, j_{r}\right)$ is only executed if it is not true. Hence we are allowed to replace the righthand side of S4 by (15).

Now some instances of $\mathrm{S} 1$ have become dead code. The instances that are still alive are given by (12) where $S=$ $\left\{S_{3}\right\}$, i.e.,

$$
\begin{aligned}
& w_{1}^{-1}\left(R_{3}\right) \cap I_{1} \\
= & w_{1}^{-1}(\{(a, b) \mid a=b+99 \wedge 0 \leq b \leq 99\}) \cap I_{1} \\
= & \{(i, j) \mid i=99 \wedge 0 \leq j \leq 99\} .
\end{aligned}
$$

Note that we left S5 out from $S$ since instances of S5 do not read elements written by $\mathrm{S} 1$ - this was exactly the intention of the propagation. Inserting the conditions not implied by the iterator bounds yields the program of Fig. 6 .

In general we can summarize the operation to do copy propagation from a statement $\mathbf{S}_{x}$ that writes to an array, to statement $\mathbf{S}_{y}$ that reads from the same array, as follows:

Algorithm 1. Non-recursive copy propagation

Goal: Propagate an assignment $\mathrm{S}_{x}$, with definition mapping $w_{x}\left(\vec{\imath}_{x}\right)=A \cdot \vec{\imath}_{x}+c$, to a statement $\mathbf{S}_{y}$, with operand mapping $r_{y}$

1. Calculate $w_{x}^{-1}$. Since $w_{x}$ is of the form of (1), its inverse is simply $w_{x}^{-1}: \vec{a} \mapsto A^{-1} \cdot(\vec{a}-\vec{c})$.

2. Calculate $\vec{\imath}_{x}=\left(w_{x}^{-1}\right)\left(r_{y}\left(\vec{\imath}_{y}\right)\right)$.

3. Calculate condition $\vec{\imath}_{x} \in I_{x}$. If all statements in the program that write to the array in question, have the same definition mapping and if they share some loops,

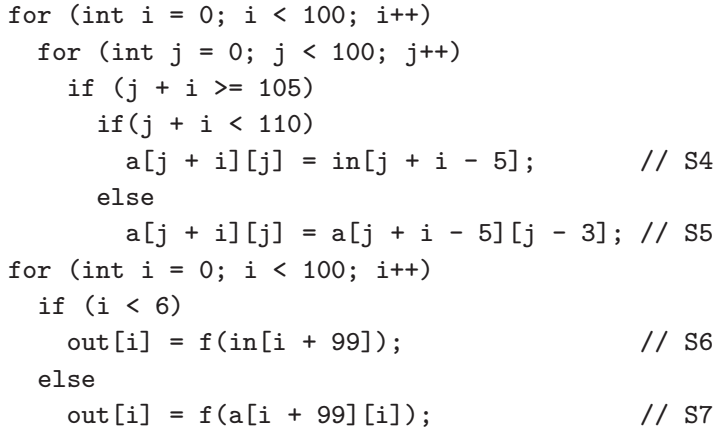

Figure 7: $\mathrm{S} 1$ propagated to $\mathrm{S} 3$ and then removed

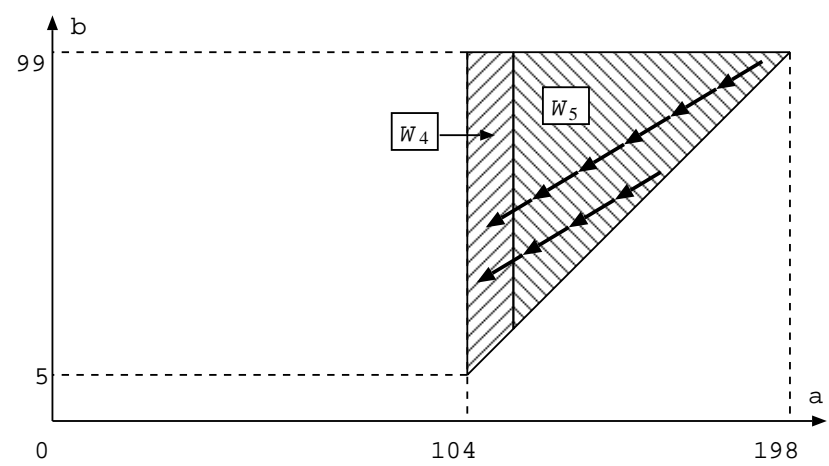

Figure 8: S5 reads array elements that it wrote itself

then remove from the condition the parts that are related to the bounds of these loops.

4. If the condition always evaluates to true (e.g., if there is just one statement that writes to the array in question), then replace the array reference in $\mathbf{S}_{y}\left(\vec{\imath}_{y}\right)$ by the righthand side of $\mathbf{S}_{x}\left(\left(w_{x}^{-1}\right)\left(r_{y}\left(\vec{\imath}_{y}\right)\right)\right)$.

5. If the condition does not always evaluate to true, split statement $\mathbf{S}_{y}$ in two statements $\mathbf{S}_{y_{1}}$ and $\mathbf{S}_{y_{2}}$ and add an if-statement such that $\mathbf{S}_{y_{1}}$ is only executed when the condition is true and $\mathbf{S}_{y_{2}}$ is executed when it is not true. Then replace the array reference in $\mathbf{S}_{y_{1}}\left(\vec{\imath}_{y}\right)$ by the righthand side of $\mathbf{S}_{x}\left(\left(w_{x}^{-1}\right)\left(r_{y}\left(\vec{\imath}_{y}\right)\right)\right)$.

6. Let $S=\left\{\mathrm{S}_{i_{1}}, \ldots, \mathrm{S}_{i_{s}}\right\}$ such that each $\mathrm{S}_{i_{j}}$ reads from the array that $\mathbf{S}_{x}$ writes to and such that $i_{j} \neq y$. Then calculate $I^{\prime}=w_{x}^{-1}\left(R_{i_{1}} \cup \ldots \cup R_{i_{s}}\right) \cap I_{x}$. If $I^{\prime}$ is empty, remove $\mathbf{S}_{x}$ from the program. Otherwise restrict the iteration space of $\mathrm{S}_{x}$ to $I^{\prime}$ by adding conditions.

Using the above algorithm we also propagate S1 to S3, resulting in the program in Fig. 7. Since S1 was now completely dead code, it has been removed.

\subsection{Recursive copy propagation}

In this section we show how to apply the basic idea of Sec. 3.1 to a statement that reads array elements that it wrote itself. An example of this in Fig. 7 is statement S5. The behaviour of this statement is schematically represented in Fig. 8. An arrow from one array element to another array element means that the first element is copied by S5 from the other array element. Only arrows are drawn for two chains of copies. From Fig. 8 we can conclude that if we want to 
remove the copy operation from S5, we would need to apply Algorithm 1 as many times as the longest copy chain, each time removing one copy operation from the chain. Doing this is not a good idea since the length of copy chains can be very large and each propagation possibly introduces an if-statement, so the program size may blow up.

Equation (14) also applies to statement $\mathbf{S} 5$, so $\mathbf{S} 5\left(i_{r}, j_{r}\right)$ reads the value written by $\mathbf{S} 5\left(i_{r}-2, j_{r}-3\right)$. Now consider a chain beginning from $\mathrm{S} 5\left(i_{r}, j_{r}\right)$ that contains $n$ recursive copy operations, i.e., $n$ arrows within $W_{5}$ followed by one arrow from $W_{5}$ to $W_{4}$. The intention is to bypass the $n$ recursive copy operations. To achieve this, the righthand side of $\mathbf{S} 5\left(i_{r}, j_{r}\right)$ needs to be replaced by the righthand side of $\mathrm{S} 5\left(i_{r}-2 \cdot n, j_{r}-3 \cdot n\right)$. To perform such a transformation, we need to find the value of $n$ (which depends on the value of the iterators $i_{r}$ and $j_{r}$ ). The iteration space of S5 is

$$
\begin{aligned}
I_{5}=\{(i, j) \mid & \leq \leq i<100 \wedge 0 \leq j<100 \\
& \left.\wedge j+i \geq 110 \wedge(i, j) \in \mathbb{Z}^{2}\right\} .
\end{aligned}
$$

$n$ is the maximal $k$ such that $\mathrm{S} 5\left(i_{r}-2 \cdot k, j_{r}-3 \cdot k\right)$ is in the iteration space $I_{5}{ }^{3}$ This means that

$$
\begin{gathered}
0 \leq i_{r}-2 \cdot k \leq 99 \wedge 0 \leq j_{r}-3 \cdot k \leq 99 \\
\wedge j_{r}+i_{r}-5 \cdot k \geq 110 .
\end{gathered}
$$

Note that we removed the strict inequalities using the fact that all variables are integer. Solving for $k$ gives

$$
\begin{gathered}
\max \left(\left(i_{r}-99\right) / 2,\left(j_{r}-99\right) / 3\right) \leq k \wedge \\
k \leq \min \left(i_{r} / 2, j_{r} / 3,\left(j_{r}+i_{r}-110\right) / 5\right) .
\end{gathered}
$$

Note that there is always a non-negative solution as $k=$ 0 expresses that $\left(i_{r}, j_{r}\right) \in I_{5}$. The value $n$ of the longest recursive copy chain is given by the maximal integer value of $k$, hence (with $\div$ being integer division)

$$
n=\min \left(i_{r} \div 2, j_{r} \div 3,\left(j_{r}+i_{r}-110\right) \div 5\right) \text {. }
$$

For $\left(i_{r}, j_{r}\right) \in I_{5}$, the minimal value is always $\left(j_{r}+i_{r}-110\right) \div 5$ so we obtain

$$
n=\left(j_{r}+i_{r}-110\right) \div 5
$$

Therefore the first statement in the copy chain that no longer reads array elements written by the statement itself is

$$
\text { S5 }\left(i_{r}-2 \cdot\left(\left(j_{r}+i_{r}-110\right) \div 5\right), j_{r}-3 \cdot\left(\left(j_{r}+i_{r}-110\right) \div 5\right)\right) \text {. }
$$

To skip the recursive copy chain we read the righthand side of this statement instance instead of the original righthand side. This results in the program of Fig. 9. Remember that division of integers is always integer division in $\mathrm{C}$.

Let us now look at the general case. We have a statement $\mathrm{S}_{x}\left(\vec{\imath}_{x}\right)$ that copies element $r_{x}\left(\vec{\imath}_{x}\right)$ to element $w_{x}\left(\vec{\imath}_{x}\right)$ of the same array. This statement also reads elements it wrote itself, i.e., $W_{x} \cap R_{x} \neq \emptyset$. A first assumption is that the operation executed by $\mathrm{S}_{x}$ is a copy operation, in contrast to Sec. 3.2 where any righthand side was allowed in the assignment we propagated. A second assumption is that $w_{x}^{-1}\left(r_{x}\left(\vec{\imath}_{x}\right)\right)$ is of the following form:

$$
w_{x}^{-1}\left(r_{x}\left(\vec{\imath}_{x}\right)\right)=\vec{\imath}_{x}+\vec{\Delta} .
$$

This means that the iteration that wrote the value read by iteration $\vec{\imath}_{x}$ is displaced by a constant vector $\vec{\Delta}$. Because of the affine indexing, this also means that the arrows in Fig. 8

${ }^{3}$ Also $\mathrm{S} 5\left(i_{r}-2 \cdot l, j_{r}-3 \cdot l\right)$ should be in $I_{5}$ for $0 \leq l \leq k$. This holds as $I_{5}$ is convex.

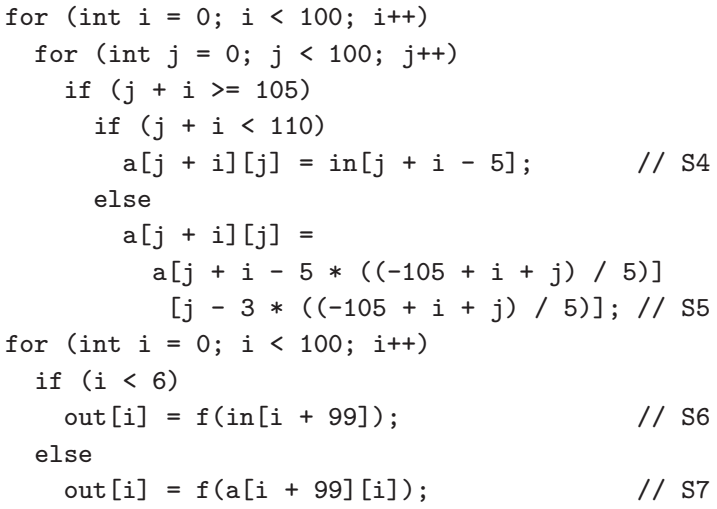

Figure 9: Solved the recursive copy propagation for S5

all have the same length and all point in the same direction. A third assumption is that the iteration domain $I_{x}$ is convex, i.e., the condition on the iterators is a conjunction (i.e., an "and") of affine inequalities. If the iteration space happens to contain a disjunction (i.e., an "or"), it can be split up into a union of convex iteration domains and each should be treated separately. If an iteration domain is convex, the inequalities governing the iterators can be concisely written as follows:

$$
A \cdot \vec{\imath}_{x}+\vec{b} \geq 0 .
$$

Here $A$ has $m$ rows, so there are $m$ inequalities. Strict inequalities (e.g., $a>b)$ are converted to non-strict inequalities (e.g., $a \geq b+1)$ where necessary. We again leave out the condition that the iterators should be integer because we work with integer variables only.

We can then, as in the example, find an appropriate value for $n$ such that statement $\mathbf{S}_{x}$ for the iteration indicated by $\vec{\imath}_{x}+n \cdot \vec{\Delta}$ no longer reads values written by $\mathrm{S}_{x}$ itself. We do this by substituting $\vec{\imath}_{x}+n \cdot \vec{\Delta}$ for $\vec{\imath}_{x}$ in (29), yielding the following:

$$
A \cdot\left(\vec{\imath}_{x}+n \cdot \vec{\Delta}\right)+\vec{b} \geq 0 \Leftrightarrow n \cdot A \cdot \vec{\Delta}+A \cdot \vec{\imath}_{x}+\vec{b} \geq 0
$$

This is a set of $m$ inequalities. Referring to the $j$ th element of a vector by a subscript $j$, these inequalities are:

$$
n \cdot(A \cdot \vec{\Delta})_{j}+\left(A \cdot \vec{\imath}_{x}\right)_{j}+\vec{b}_{j} \geq 0 \text { with } 1 \leq j \leq m
$$

We are only interested in the inequalities that provide an upper bound on $n$, i.e. the ones for which $(A \cdot \vec{\Delta})_{j}$ is negative. We group the index of these inequalities in the set

$$
J_{-}=\left\{j \mid 1 \leq j \leq m \wedge(A \cdot \vec{\Delta})_{j}<0\right\}
$$

The upper bound on $n$ then becomes:

$$
n \leq \min _{j \in J_{-}}\left(\left(\left(A \cdot \vec{\imath}_{x}\right)_{j}+\vec{b}_{j}\right) \div\left(-(A \cdot \vec{\Delta})_{j}\right)\right)
$$

Note that the transformed statement is not suited for further recursive copy propagation unless it can be decided which element is the minimum one (this was the case in our example) and the integer division can be simplified, i.e., when $(A \cdot \Delta)_{j}=-1$ (this was not the case in our example). If all integer divisions can be simplified, we can still make the statement suited for further recursive copy propagation by 


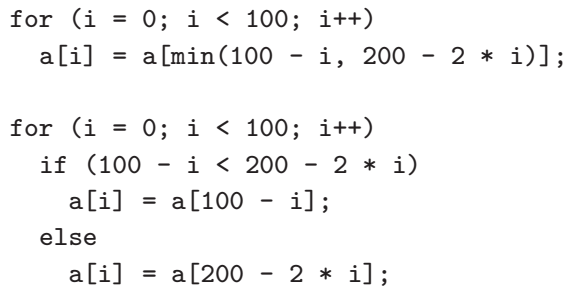

Figure 10: With (top) and without (bottom) minimum operation

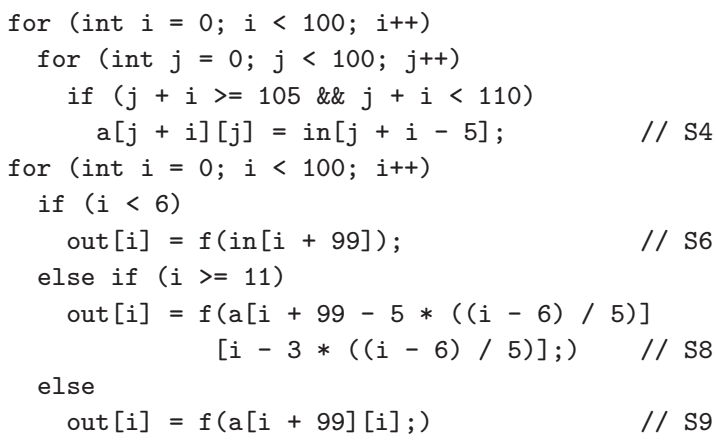

Figure 11: S5 propagated to S7 and then removed

eliminating the minimum function as illustrated in Fig. 10. ${ }^{4}$ The algorithm for solving the recursion then becomes:

\section{Algorithm 2. Recursive copy propagation}

Goal: Solve a recursive copy chain in $\mathbf{S}_{x}$ with $w_{x}$ and $r_{x}$ as respective definition and operand mappings

1. Calculate $w_{x}^{-1}\left(r_{x}\left(\vec{\imath}_{x}\right)\right)$ and find the vector $\vec{\Delta}$ according to (28) ; exit if $\vec{\Delta}$ is not constant.

2. Write the inequalities on the iterators as $A \cdot \vec{\imath}_{x}+\vec{b} \geq 0$. Let $m$ be the number of rows of $A$.

3. Calculate $n$ using (32) and (33).

4. Replace the righthand side of $\mathrm{S}_{x}\left(\vec{\imath}_{x}\right)$ by the righthand side of $\mathrm{S}_{x}\left(\vec{\imath}_{x}+n \cdot \vec{\Delta}\right)$.

5. If enabling further recursive copy propagation, then remove the minimum operation as shown in Fig. 10.

6. Let $S=\left\{\mathrm{S}_{i_{1}}, \ldots, \mathrm{S}_{i_{s}}\right\}$ such that $\mathrm{S}_{i_{j}}$ reads from the array $\mathrm{S}_{x}$ writes to and such that $i_{j} \neq x$. Then calculate $I^{\prime}=w_{x}^{-1}\left(R_{i_{1}} \cup \ldots R_{i_{s}}\right) \cap I_{x}$. If $I^{\prime}$ is empty, remove $\mathrm{S}_{x}$ from the program. Otherwise restrict the iteration space of $\mathrm{S}_{x}$ to $I^{\prime}$ by adding conditions.

\subsection{Wrapping up the example}

Since the recursive copy chain has been removed in Fig. 9, we can now propagate statement S5 to S7 using Algorithm 1 and then remove S5 from the program since it is dead code. The result is shown in Fig. 11. In a final step, S4 is propagated to S8 and S9, again using Algorithm 1, and then removed. This is shown in Fig. 12. The final result is a

${ }^{4}$ By the nature of the transformation, the transformed statement is not a candidate for recursive copy propagation, but after some non-recursive copy propagation steps, the righthand side can appear in a statement that is a candidate for recursive copy propagation.

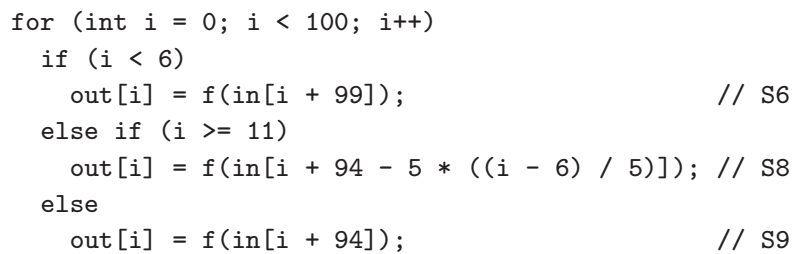

Figure 12: S4 propagated to $S 9$ and then removed

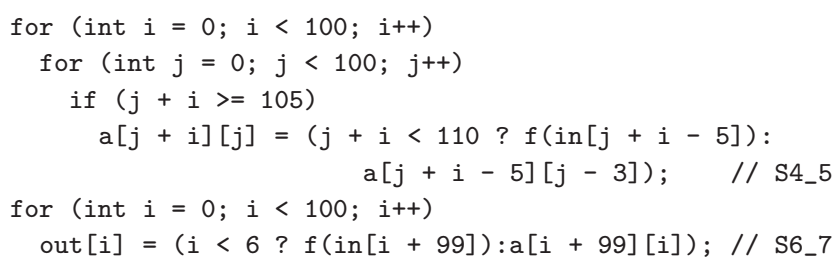

Figure 13: Grouping of statements using the ? operator from $\mathrm{C}$

fairly simple program compared to the intermediate results ${ }^{5}$. This example has however been engineered to keep the calculations relatively simple, but it can nonetheless show the strength of our approach. In practice this is not necessarily so, e.g. for the multimedia kernels from our experiments.

\subsection{Global algorithm}

In this section we use the two basic operations of Sec. 3.2 and 3.3 to eliminate copy operations, i.e., statements with a single array reference as righthand side. Our methods apply to assignments that involve some computation in the righthand side as well. However when eliminating copy operations, the number of memory accesses can never increase since we replace each runtime memory access by another one. ${ }^{6}$ In case of a general righthand side, this is not necessarily so and this will not be handled in this paper.

Before applying advanced copy propagation, we will change the representation of the subject program slightly. This consists of grouping copy operations that have an identical lefthand side and that are adjacent in the program and in the same loop nest. E.g., for the program in Fig. 7, this would look like the program in Fig. 13. We use a convenient notation from $\mathrm{C}$ here. The reason for grouping these statements is that we can then propagate a whole group of copy operations at once, having to do calculations only once for the whole group instead of separately for each copy operation in it. Hence in Fig. 13, only one call to Algorithm 1 is needed to propagate $\mathbf{S 4} 5$ to $\mathbf{S 6 \_ 7}$ while doing the equivalent propagations in Fig. 7 would require two calls to the algorithm. Furthermore we suppose the subject program was converted to DSA form as in [6] or [9], or by the DSA conversion we are investigating ourselves. In this case the lefthand side of all assignments to a certain array have the

${ }^{5}$ The attentive reader has probably noticed that statement S8 and S9 can be melted together, resulting in an even simpler program. This is so because S9 is only executed for $i$ between 6 and 10, and then $(i-6) / 5$ simplifies to 0 . The reader can check that if we first solved the recursion in S2 before doing any copy propagation, then we would get the version with S8 and S9 merged.

${ }^{6}$ Note that this is not necessarily true for textual memory accesses because of statement splitting, cf. Fig. 10. 


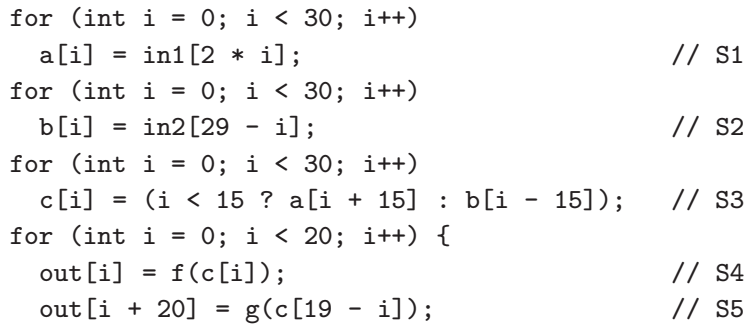

Figure 14: The order of propagations can be important

same definition mapping, enabling us to group all copy operations to a certain array in a single copy operation.

The general idea behind the algorithm is to first remove all recursive copy operations that are present in the program, if they fulfill all preconditions to Algorithm 2. After that we will propagate all non-recursive copy operations to an array to wherever the array is read. This can create new recursive copy operations (that were indirect in the original program, e.g., a statement that copies array elements written by another statement, and vice versa.) So we repeat both steps as long as they can be applied.

For the second step we would like to be cautious concerning the order in which the non-recursive propagations are done. If we look at the program in Fig. 14, we can see that if we first propagate $\mathrm{S} 1$ and $\mathrm{S} 2$ to S3 and then S3 to S4 and $\mathrm{S} 5$, then we need to apply Algorithm 1 four times. If we however first propagate S3 to both S4 and S5, then in S4 and S5 will appear references to both arrays a and b, meaning we have to propagate S1 and S2 to both S4 and S5, and hence we get a total of six calls to Algorithm 1. In longer copy chains, the difference in the number of times Algorithm 1 needs to be applied can grow exponentially. The idea is then to only propagate a copy operation to a read if all reads in the copy operation have been propagated to. We should however watch out for recursive chains of copy operations, e.g., a statement that copies elements written by another statement and vice versa. In the algorithm described below this case is caught using a visit variable associated with each statement and one copy operation in the recursive chain is propagated although its reads have not been propagated to.

Finally another touch of caution is taken in case not all recursive copy operations can be removed. In this case we do not propagate this copy operation to any read because the recursion will cause the read to still be from an array element written by the recursive copy operation and hence we would have to apply our algorithm as many times as the length of the copy chain, and the complexity of the resulting program would grow as many times as well.

\section{Algorithm 3. Advanced copy propagation}

Goal: Propagate as many copy operations as possible in a program $P$ in DSA form with all copy operations to a certain array grouped together

Repeat

1. For each statement $S$ in $P$, for each recursion in $S$, apply Algorithm 2

2. For each statement $S$ in $P$, S.visit $\leftarrow$ NOTVISITED

3. For each statement $S$ in $P$, call
NonRecursiveCopyProgation $(S, P)$

Until there is no more change

NonRecursiveCopyPropagation $(S, P)$ begin

1. If $S$.visit $=$ VISITING then return;

2. If S.visit $\neq$ VISITED

(a) S.visit $\leftarrow$ VISITING

(b) For each read operation in $S$, find the copy statement $S^{\prime}$ in $P$ that writes to the array the read operation reads from, and call NonRecursiveCopyPropagation $\left(S^{\prime}, P\right)$

(c) S.visit $\leftarrow$ VISITED

3. For each read operation in $S$, find the copy statement $S^{\prime}$ in $P$ that writes to the array the read operation reads from, and use Algorithm 1 to propagate $S^{\prime}$ to $S$ end

Although Algorithm 3 loops until a fixed point is reached, we can find an upper limit for the number of times both Algorithm 1 and Algorithm 2 are called from Algorithm 3. Note that each recursion is solved at most once, so if the number of direct and indirect recursions in the program is $r$, then Algorithm 2 is called $O(r)$ times.

Also note that each non-recursive copy statement is only propagated once to each read operation. By our depth-first traversal of the chains of copy operations, we make sure that every read is propagated to before it is propagated to another read, so if we propagate a copy operation to a read, the reads with which it will be replaced will not be propagated to anymore. Hence if the number of reads from arrays that are copies from other arrays is $c$, then the number of times Algorithm 1 is called is $O(c)$. There is one exception though, namely when there is an indirect recursion. If the maximum number of copy statements that get grouped together is $k$, then a maximum of $O(r \cdot k)$ reads will be duplicated, and each can be propagated to. So Due to recursive copy chains there can be an extra of $O(r \cdot k)$ calls to Algorithm 1, giving a total of $O(c+r \cdot k)$ calls to Algorithm 1 .

As for the complexity of Algorithm 1 and 2, we note that the polyhedral operations only depend on the dimension of the polyhedra operated on, which is the number of loops that are around a statement. Since the depth of loop nests is generally limited (highest we have ever observed is 10 which is a real rarity), we can safely assume that the execution time of the polyhedral operations is bounded by a constant. For Algorithm 2 the number of polyhedral operations is constant, so Algorithm 2 executes in constant time. Since there are $O(r)$ calls to Algorithm 2, all executions of it take a time that is $O(r)$. Algorithm 1 however executes a variable number of polyhedral calculations, since each of the copy operations that are grouped in the righthand side of the copy operation need to be adjusted before substituting it for the read we propagate to. Thus the execution time of one call to Algorithm 1 is $O(k)$. Since Algorithm 1 is called $O(c+r \cdot k)$ times, all executions of it take a time that is $O\left(k \cdot c+r \cdot k^{2}\right)$.

Thus we find $O\left((r)+\left(k \cdot c+r \cdot k^{2}\right)\right)$ as the total execution time for Algorithm 3. Note that $k$ does not grow large either in most cases, so it can be bounded by a constant. This bound can be guaranteed by only allowing propagations that do not make the result go above that bound. The 


\begin{tabular}{|c|c|c|c|c|c|c|c|}
\hline Name & $\Delta a c c$ & $\Delta a c c_{r}$ & $\Delta m e m$ & Alg1 & Alg2 & $c$ & $t(\mathrm{~s})$ \\
\hline LU1 & $32 \%$ & $26 \%$ & $40 \%$ & 8 & 1 & 6 & 0.191 \\
\hline LU2 & $37 \%$ & $26 \%$ & $45 \%$ & 8 & 0 & 8 & 0.332 \\
\hline im1 & $44 \%$ & $43 \%$ & $56 \%$ & 6 & 0 & 6 & 0.367 \\
\hline im2 & $95 \%$ & $94 \%$ & $100 \%$ & 6 & 0 & 6 & 1.700 \\
\hline mp3 & $30 \%$ & $22 \%$ & $37 \%$ & 13 & 2 & 13 & 0.929 \\
\hline divx & $31 \%$ & $31 \%$ & $100 \%$ & 1 & 0 & 1 & 0.134 \\
\hline
\end{tabular}

Table 1: Results of advanced copy propagation

complexity then becomes $O(c+r)$, so it is linear in the number of recursions and the number of reads from arrays that are copied from others. Hence the method should scale well.

\section{EXPERIMENTAL RESULTS}

Algorithm 1, 2 and 3 described above have been implemented using [15] as polyhedral library. The codes we have tested our implementation on have been manually transformed to DSA form and have been made pointer-free by translating pointer references to array references. Also due to current parser restrictions, all irrelevant code was removed so the remaining codes are the computational kernels only. The size of these kernels ranges from 29 to 91 lines of $\mathrm{C}$-code. The results are shown in Table 1. $\Delta$ acc refers to the decrease in memory accesses (both reads and writes) between the DSA version and the propagated version of the programs. Since DSA conversion was done by hand, we introduced copy operations to make it easier and less error-prone. Hence we also list $\Delta a c c_{r}$ which gives the "real" decrease in memory accesses between the original and the propagated version. This decrease in memory accesses is important because the power consumption of memories grows as the number of accesses increases. $\Delta$ mem stands for the decrease in memory size given that the program stays in DSA form. The memory size can change when translated out of DSA form, so the number is only indicative rather than an exact measure. Then Alg1 and Alg2 are respectively the number of times Algorithm 1 and Algorithm 2 are called. The number of reads from arrays that are copies is represented by $c$ - see the complexity analysis - and $t$ is the execution time of the advanced copy propagation algorithm.

LU1 and LU2 are two equivalent pieces of code from part of LU-factorisation. Note the differences between the two. First it is to be noted that in both cases all copy operations were removed and the same set of operations remained, so memory use and accesses after propagation were the same. However in LU2 more memory accesses and memory elements were removed. The difference lies in copy operations introduced by conversion to DSA, as can be observed in the equal value for $\Delta a c c_{r}$. The fact that there were more copy operations added in LU2 suggests that the data flow is more complex there, which results in more complex righthand sides. Hence the number of statements grouped together becomes comparable to $c$ leading to a higher execution time. Also, the number of times Algorithm 1 was called for LU1 was higher than $c$, caused by the presence of a recursion.

Both im1 and im2 are examples from image processing, where operations like scaling and rotation are present, which are inherently copy operations. im1 contains anti-aliasing code which prohibits propagation, but im2 contains no antialiasing code and hence all intermediate copy operations can be removed. This results in a complex righthand side, which is reflected in the execution time.

mp3 is the subband synthesis code from an mp3-encoder. Note that although it contained two recursions, it didn't lead to more calls to Algorithm 1 than necessary, as indicated by c. The last example is the repetitive image padding code from a divx-codec. This is an example where a border was added to the image to ease the task of programming, but this border was completely removed as can be seen by the percentages.

\section{TO DSA OR NOT TO DSA}

In this paper we described techniques for applying copy propagation on programs in DSA form. A consequence of our choice of using DSA is that our techniques are rather simple and elegant. We need a data-flow analysis, just like many other compiler optimizations, but in our case it boils down to calculating a simple matrix inverse. On non-DSA code, we would need the complex data-flow analysis from [6]. Also as shown in Sec. 3.1 we can always do copy propagation in a DSA program, while for classic copy propagation certain conditions have to be met. Hence copy propagation on non-DSA programs would leave many opportunities to do optimizations unused.

The advantages of DSA are not limited to copy propagation only. Also the loop transformations described in [4] are simpler and have more freedom when applied to DSA programs. Since each array element is just written once, the only constraint is that no read from an array element should be executed before the write to it. If this was not so, additional constraints would be that a read must be executed before the value to be read is overwritten.

In [3] many more transformations are outlined for optimizing embedded applications, like introducing smaller (and hence less energy-consuming) memories containing data that is heavily used. Many of these optimizations can do a much better job on DSA code, using simpler techniques.

However transforming to DSA blows up memory use. If the original program contained an array of 10 elements, and each of these was assigned 100 times, the DSA version would need at least 1000 elements because there are 1000 assignments. Methods exist that compact these arrays [5, 14, 17]. Again, these can do a much better job on DSA-code, using simpler methods, especially when assisted by loop transformations that increase data locality [4]. In [3], DSA form is used along the whole optimization trajectory for embedded systems and very good results are attained.

At the moment only a subset of C-programs can be converted to DSA by methods like $[6,9]$. However we are currently researching a method to extend the subset that can be transformed to DSA, hoping to be able to handle the most common constructs. Furthermore we can apply the algorithms in this paper to those parts of the code that can be transformed to DSA and leave the rest as is.

\section{DISCUSSION}

In this paper we have introduced non-recursive and recursive copy propagation and have used these as basic operations to implement a method to do advanced copy propagation over global loop and condition scopes of imperative programs in dynamic single assignment form. We have tested this prototype implementation on a few selected multimedia codes. Currently any chance for propagation is taken, but this introduces extra code. However it is a well-known fact 
that a memory access is more expensive than other operations, so it seems plausible to add extra code as long as we spare memory accesses. A more important concern is that code becomes more complicated, which can be a problem for subsequent optimizations, but is also pretty bad for the code cache in an embedded system. However the method can be adapted to do selective copy propagation, but this requires steering. This steering is part of future research.

We apply our methods to programs in dynamic single assignment form. This DSA form is also widely used in the context of parallelization [7] and systolic array design [11]. In [6], Feautrier presents an automated method for dynamic single assignment conversion, but it is limited in both applicability and scalability. We are currently working on a new method that tries to overcome those limitations.

The goals of our methods are similar to those of copy propagation and constant propagation $[1,12]$ that have been used for a long time in compilers. They often limit themselves to simple data types. In [1] an extension to arrays is presented. A dependence analysis between array references is required but is left unspecified in [1]. In [12] Muchnick gives a number of dependence tests, ranging from Banerjee's GCD test[2] to Pugh's Omega test[13], which allow the data-flow analysis required for copy propagation to be made more accurate for array accesses. An extension to constant propagation that takes conditional branches into account is given by Wegman and Zadeck[18], but it is limited to branch conditions that evaluate to a constant true or false. To the best of our knowledge, our work is the first attempt to automate copy propagation and constant propagation for programs with arrays by treating each instance of each statement separately, and thus allowing array propagation on an element basis. Each of the previously existing methods for copy and constant propagation consider all instances of a statement as a whole and hence they do these transformations on an all-or-nothing basis. We can also propagate over conditional statements and global loops that often hinder classic copy and constant propagation.

The intention of doing classic copy propagation is to create dead code that can then be eliminated. Dead-code elimination is described in $[1,12]$. Here too compilers often limit themselves to simple data types, while technically the same dependence tests can increase the precision in case of arrays. Partial dead-code elimination is presented in [10] and allows to remove code that is dead on only part of the program paths by moving it down the paths along which the code is not dead. However the code is textually moved without changing the branching structure of the program. Again to the best of our knowledge, we are the first to automate dead-code elimination on a statement instance basis.

The further development of our prototype, together with its integration in the other steps in the framework of [3] will allow us to measure the effects of advanced copy propagation on large multimedia appications.

\section{REFERENCES}

[1] A. V. Aho, R. Sethi, and J. D. Ullman. Compilers: Principles, Techniques and Tools. Addison-Wesley, Inc., 1986.

[2] U. Banerjee. Dependence testing in ordinary programs. Master's thesis, Dept. of Comp. Sci., Univ. of Illinois, Nov. 1976.
[3] F. Catthoor, S. Wuytack, E. De Greef, F. Balasa, L. Nachtergaele, and A. Vandecappelle. Custom Memory Management Methodology: Exploration of Memory Organisation for Embedded Multimedia System Design. Kluwer Academic Publishers, 1998.

[4] K. Danckaert. Loop transformations for data transfer and storage reduction on multiprocessor systems. $\mathrm{PhD}$ thesis, K.U.Leuven, 2001.

[5] E. De Greef, F. Catthoor, and H. De Man. Memory size reduction through storage order optimization for embedded parallel multimedia applications. In Proceedings of the Workshop on Parallel Processing and Multimedia of the International Parallel Processing Symposium, pages 84-98, 1997.

[6] P. Feautrier. Dataflow analysis of array and scalar references. International Journal of Parallel Programming, 20(1):23-53, 1991.

[7] M. W. Hall, J. M. Anderson, S. P. Amarasinghe, B. R. Murphy, S.-W. Liao, E. Bugnion, and M. S. Lam. Maximizing multiprocessor performance with the suif compiler. In IEEE Computer, December 1996.

[8] J. Hennessy and D. Patterson. Computer Architecture: A Quantitative Approach. Morgan Kaufmann Publishers, San Francisco, CA, 1996.

[9] B. Kienhuis. Matparser: An array dataflow analysis compiler. Technical report, University of California, Berkeley, February 2000.

[10] J. Knoop, O. Ruthing, and B. Steffen. Partial dead code elimination. In SIGPLAN Conference on Programming Language Design and Implementation, pages 147-158, 1994.

[11] H. Kung and C. Leiserson. Systolic arrays for VLSI. In Sparse Matrix Proceedings, pages 245-282, Philadelphia:SIAM, 1978.

[12] S. Muchnick. Advanced compiler design 83 implementation. Morgan Kaufmann Publishers, San Francisco, CA, 1997.

[13] W. Pugh. The Omega test: A fast and practical integer programming algorithm for dependence analysis. In Proceedings of Supercomputing '91, Albuquerque, NM, 1991.

[14] F. Quilleré and S. Rajopadhye. Optimizing memory usage in the polyhedral model. ACM Transactions on Programming Languages, 22(5):773-815, September 2000.

[15] P. Quinton, S. Rajopadhye, and T. Risset. On manipulating $\mathbb{Z}$-polyhedra. Technical report, Institut de Recherche en Informatique et Systemes Aleatoires, 1996.

[16] L. Thiele and U. Artz. On the synthesis of massively parallel architectures. International journal of high speed electronics and systems, 4(2):99-131, 1993.

[17] R. Tronçon, M. Bruynooghe, G. Janssens, and F. Catthoor. Storage size reduction by in-place mapping of arrays. In A. Cortesi, editor, Verification, Model Checking and Abstract Interpretation, Third Int. Workshop, VMCAI 2002, Revised Papers, volume 2294 of $L N C S$, pages 167-181. Springer-Verlag, 2002.

[18] M. Wegman and K. Zadeck. Constant propagation with conditional branches. ACM Transactions on Programming Languages and Systems, 13(2):181-210, April 1991. 